\title{
On Properties of a Regular Simplex Inscribed into a Ball
}

${ }^{1}$ P. G. Demidov Yaroslavl State University, 14 Sovetskaya str., Yaroslavl 150003, Russia.

MSC2020: 41A05, 52B55, 52C07

Research article

Full text in Russian

Let $B$ be a Euclidean ball in $\mathbb{R}^{n}$ and let $C(B)$ be a space of continuos functions $f: B \rightarrow \mathbb{R}$ with the uniform norm $\|f\|_{C(B)}:=$ $\max _{x \in B}|f(x)|$. By $\Pi_{1}\left(\mathbb{R}^{n}\right)$ we mean a set of polynomials of degree $\leq 1$, i. e., a set of linear functions upon $\mathbb{R}^{n}$. The interpolation projector $P: C(B) \rightarrow \Pi_{1}\left(\mathbb{R}^{n}\right)$ with the nodes $x^{(j)} \in B$ is defined by the equalities $\operatorname{Pf}\left(x^{(j)}\right)=f\left(x^{(j)}\right), j=1, \ldots, n+1$.The norm of $P$ as an operator from $C(B)$ to $C(B)$ can be calculated by the formula $\|P\|_{B}=\max _{x \in B} \sum\left|\lambda_{j}(x)\right|$. Here $\lambda_{j}$ are the basic Lagrange polynomials corresponding to the $n$-dimensional nondegenerate simplex $S$ with the vertices $x^{(j)}$. Let $P^{\prime}$ be a projector having the nodes in the vertices of a regular simplex inscribed into the ball. We describe the points $y \in B$ with the property $\left\|P^{\prime}\right\|_{B}=\sum\left|\lambda_{j}(y)\right|$. Also we formulate some geometric conjecture which implies that $\left\|P^{\prime}\right\|_{B}$ is equal to the minimal norm of an interpolation projector with nodes in $B$. We prove that this conjecture holds true at least for $n=1,2,3,4$.

Keywords: simplex; ball; linear interpolation; projector; norm

\section{INFORMATION ABOUT THE AUTHORS} \begin{tabular}{r|l} 
Mikhail Viktorovich Nevskii & orcid.org/0000-0002-6392-7618. E-mail: mnevsk55@yandex.ru \\
correspondence author & Head of Department, Doctor of Science, Docent.
\end{tabular}

For citation: M. V. Nevskii, “On Properties of a Regular Simplex Inscribed into a Ball”, Modeling and analysis of information systems, vol. 28, no. 2, pp. 186-197, 2021. 


\section{О свойствах правильного симплекса, вписанного в шар}

М. В. Невский ${ }^{1}$

DOI: $10.18255 / 1818-1015-2021-2-186-197$

${ }_{1}^{1}$ Ярославский государственный университет им. П. Г. Демидова, ул. Советская, д. 14, г. Ярославль, 150003 Россия.

УДК $514.17,517.51,519.6$

Научная статья

Полный текст на русском языке
Получена 28 апреля 2021 г.

После доработки 25 мая 2021 г. Принята к публикации 26 мая 2021 г.

Пусть $B$ - евклидов шар в $\mathbb{R}^{n}, C(B)$ - пространство непрерывных функций $f: B \rightarrow \mathbb{R}$ с равномерной нормой $\|f\|_{C(B)}:=\max _{x \in B}|f(x)|$. Под $\Pi_{1}\left(\mathbb{R}^{n}\right)$ понимается совокупность многочленов от $n$ переменных степени $\leq 1$, то есть линейных функций на $\mathbb{R}^{n}$. Интерполяционный проектор $P: C(B) \rightarrow \Pi_{1}\left(\mathbb{R}^{n}\right)$ с узлами $x^{(j)} \in B$ определяется равенствами $P f\left(x^{(j)}\right)=f\left(x^{(j)}\right), j=1, \ldots, n+1$. Норма $P$ как оператора из $C(B)$ в $C(B)$ вычисляется по формуле $\|P\|_{B}=$ $\max _{x \in B} \sum\left|\lambda_{j}(x)\right|$, где $\lambda_{j}$ - базисные многочлены Лагранжа невырожденного $n$-мерного симплекса с вершинами $x^{(j)}$. Пусть $P^{\prime}$ - проектор, узлы которого совпадают с вершинами правильного симплекса, вписанного в шар. В статье найдены точки $y \in B$, для которых $\left\|P^{\prime}\right\|_{B}=\sum\left|\lambda_{j}(y)\right|$. Формулируется геометрическая гипотеза, из справедливости которой следует, что $\left\|P^{\prime}\right\|_{B}$ есть минимальное значение нормы интерполяционного проектора, узлы которого принадлежат $B$. Доказывается, что эта гипотеза справедлива по крайней мере для $n=1,2,3,4$.

Ключевые слова: симплекс; шар; линейная интерполяция; проектор; норма

\section{ИНФОРМАЦИЯ ОБ АВТОРАХ}

Михаил Викторович Невский | orcid.org/0000-0002-6392-7618. E-mail: mnevsk55@yandex.ru автор для корреспонденции

Для цитирования: М. V. Nevskii, “On Properties of a Regular Simplex Inscribed into a Ball”, Modeling and analysis of information systems, vol. 28, no. 2, pp. 186-197, 2021.

( Невский М. В., 2021

Эта статья открытого доступа под лицензией СС BY license (https://creativecommons.org/licenses/by/4.0/). 


\section{Введение}

Пусть $\Omega$ - выпуклое тело в $\mathbb{R}^{n}$. Обозначим через $C(\Omega)$ пространство непрерывных функций $f: \Omega \rightarrow \mathbb{R}$ с равномерной нормой

$$
\|f\|_{C(\Omega)}:=\max _{x \in Q_{n}}|f(x)| .
$$

Под $\Pi_{1}\left(\mathbb{R}^{n}\right)$ будем понимать совокупность многочленов от $n$ переменных степени не выше 1 , или, иначе говоря, линейных функций на $\mathbb{R}^{n}$. Для $x^{(0)} \in \mathbb{R}^{n}, R>0$ через $B\left(x^{(0)} ; R\right)$ обозначим $n$-мерный евклидов шар, задаваемый неравенством $\left\|x-x^{(0)}\right\| \leq R$. Здесь

$$
\|x\|:=\sqrt{(x, x)}=\left(\sum_{i=1}^{n} x_{i}^{2}\right)^{1 / 2} .
$$

Положим $B_{n}:=B(0 ; 1)$. Ниже $e_{1}, \ldots, e_{n}-$ канонический базис $\mathbb{R}^{n}$.

Пусть $S$ - невырожденный симплекс в $\mathbb{R}^{n}$. Обозначим вершины $S$ через $x^{(j)}=\left(x_{1}^{(j)}, \ldots, x_{n}^{(j)}\right)$, $1 \leq j \leq n+1$. Введём в рассмотрение следующую матрииу вершин этого симплекса:

$$
\mathrm{S}:=\left(\begin{array}{cccc}
x_{1}^{(1)} & \ldots & x_{n}^{(1)} & 1 \\
x_{1}^{(2)} & \ldots & x_{n}^{(2)} & 1 \\
\vdots & \vdots & \vdots & \vdots \\
x_{1}^{(n+1)} & \ldots & x_{n}^{(n+1)} & 1
\end{array}\right) .
$$

Пусть $\mathrm{S}^{-1}=\left(l_{i j}\right)$. Линейные многочлены $\lambda_{j}(x)=l_{1 j} x_{1}+\ldots+l_{n j} x_{n}+l_{n+1, j}$, коэффициенты которых составляют столбцы матрицы $\mathrm{S}^{-1}$, обладают свойством $\lambda_{j}\left(x^{(k)}\right)=\delta_{j}^{k}$. Мы называем $\lambda_{j}$ базисными многочленами Лагранжа, соответствующими $S$. Для $x \in \mathbb{R}^{n}$ выполняются равенства

$$
x=\sum_{j=1}^{n+1} \lambda_{j}(x) x^{(j)}, \quad \sum_{j=1}^{n+1} \lambda_{j}(x)=1 .
$$

Эти равенства означают, что числа $\lambda_{j}(x)$ являются барицентрическими координатами точки $x$. Подробнее см., например, [1, §1.1].

Будем говорить, что интерполяционный проектор $P: C(\Omega) \rightarrow \Pi_{1}\left(\mathbb{R}^{n}\right)$ соответствует симплексу $S \subset \Omega$, если его узлы совпадают с вершинами этого симплекса. Проектор $P$ определяется равенствами $P f\left(x^{(j)}\right)=f\left(x^{(j)}\right)$. Справедлив следующий аналог интерполяционной формулы Лагранжа:

$$
P f(x)=\sum_{j=1}^{n+1} f\left(x^{(j)}\right) \lambda_{j}(x)
$$

Обозначим через $\|P\|_{\Omega}$ норму $P$ как оператора из $C(\Omega)$ в $C(\Omega)$. Из (1) следует, что

$$
\|P\|_{\Omega}=\max _{x \in \Omega} \sum_{j=1}^{n+1}\left|\lambda_{j}(x)\right| .
$$

Поскольку $\lambda_{j}(x)$ суть барицентрические координаты точки $x$, имеем также

$$
\|P\|_{\Omega}=\max \left\{\sum_{j=1}^{n+1}\left|\beta_{j}\right|: \quad \sum_{j=1}^{n+1} \beta_{j} x^{(j)} \in \Omega, \quad \sum_{j=1}^{n+1} \beta_{j}=1\right\} .
$$


Пусть $\Omega=B:=B\left(x^{(0)} ; R\right)$. Как доказано в [2],

$$
\|P\|_{B}=\max _{f_{j}= \pm 1}\left[R\left(\sum_{i=1}^{n}\left(\sum_{j=1}^{n+1} f_{j} l_{i j}\right)^{2}\right)^{1 / 2}+\left|\sum_{j=1}^{n+1} f_{j} \lambda_{j}\left(x^{(0)}\right)\right|\right] .
$$

Если $S$ - правильный симплекс, вписанный в шар, то $\|P\|_{B}$ не зависит ни от центра $x^{(0)}$, ни от радиуса $R$ шара, ни от выбора такого симплекса. В этом случае (см. [2, теорема 2])

$$
\|P\|_{B}=\max \{\psi(a), \psi(a+1)\} .
$$

Здесь

$$
\psi(t):=\frac{2 \sqrt{n}}{n+1}(t(n+1-t))^{1 / 2}+\left|1-\frac{2 t}{n+1}\right|, \quad 0 \leq t \leq n+1,
$$

$a:=\left\lfloor\frac{n+1}{2}-\frac{\sqrt{n+1}}{2}\right\rfloor$.

Геометрические оценки в полиномиальной интерполяции изложены в монографии автора [1]. В частности, там приведены результаты по линейной интерполяции на единичном кубе $Q_{n}:=$ $[0,1]^{n}$, часть из которых имеет окончательный характер. Некоторые оценки для конкретных $n$ были позднее улучшены (см., например, $[3,4])$. Интерполяция линейными функциями на евклидовом шаре в $\mathbb{R}^{n}$ и смежные вопросы рассматривались в [5, 6], [2].

В настоящей статье мы дополним результаты, полученные в [2], для правильного симплекса, вписанного в шар. В пункте 1 указываются точки, в которых функция $\lambda(x):=\sum\left|\lambda_{j}(x)\right|$ достигает своего максимума на шаре. В каждой такой точке $y \in B$

$$
\lambda(y)=\sum_{j=1}^{n+1}\left|\lambda_{j}(y)\right|=\|P\|_{B}=\max \{\psi(a), \psi(a+1)\} .
$$

Эти точки располагаются на граничной сфере. Их количество равно $N=\left(\begin{array}{c}n+1 \\ k\end{array}\right)$, где $k$ совпадает с тем из чисел $a$ и $a+1$, на котором $\psi(t)$ принимает большее значение. Очевидно, что $N$ есть число $(k-1)$ мерных граней невырожденного $n$-мерного симплекса. В пункте 2 обсуждаются вопросы, связанные с инвариантностью нормы интерполяционного проектора при аффинном преобразовании. В пункте 3 формулируется геометрическая гипотеза, из справедливости которой следует минимальность нормы проектора с узлами в вершинах правильного вписанного симплекса.

\section{1. Точки максимума функции $\lambda(x)$ для правильного вписанного симплекса}

Положим для натурального $n$

$$
k=k(n):=\left\{\begin{array}{cl}
a+1, & \text { если } \psi(a+1) \geq \psi(a), \\
a, & \text { если } \psi(a+1)<\psi(a) .
\end{array}\right.
$$

Подробный анализ и таблица, содержащая первые значения $n$ и $k$, приведены в [2]. В таблице 1 эти данные дополняются числами $N=\left(\begin{array}{c}n+1 \\ k\end{array}\right)$.

При $n=1,2,3$ выполняется $k=1$. Если $n>3$, то $\sqrt{n+1}>2$, поэтому

$$
a+1=\left\lfloor\frac{n+1}{2}-\frac{\sqrt{n+1}}{2}\right\rfloor+1 \leq \frac{n+1}{2}-\frac{\sqrt{n+1}}{2}+1<\frac{n+1}{2} .
$$

Значит, при $n>3$ верно $k<\frac{n+1}{2}$. Так как $k-$ целое, то при всех $n \geq 2$ имеем $k \leq \frac{n}{2}$.

Пусть $S-n$-мерный правильный симплекс, вписанный в $n$-мерный шар $B, \lambda_{j}-$ базисные многочлены Лагранжа этого симплекса, $P: C(B) \rightarrow \Pi_{1}\left(\mathbb{R}^{n}\right)$ - соответствующий интерполяционный проектор. 
Table 1. The numbers $n, k$, and $N=\left(\begin{array}{c}n+1 \\ k\end{array}\right)$

Таблица 1. Числа $n, k и N=\left(\begin{array}{c}n+1 \\ k\end{array}\right)$

\begin{tabular}{|c|c|c|}
\hline$n$ & $k$ & $N=\left(\begin{array}{c}n+1 \\
k\end{array}\right)$ \\
\hline 1 & 1 & 2 \\
\hline 2 & 1 & 3 \\
\hline 3 & 1 & 4 \\
\hline 4 & 1 & 15 \\
\hline 5 & 2 & 21 \\
\hline 6 & 2 & 56 \\
\hline 7 & 3 & 84 \\
\hline 8 & 3 & 120 \\
\hline 9 & 3 & 330 \\
\hline 10 & 4 & 495 \\
\hline 11 & 4 & 1287 \\
\hline 12 & 5 & 2002 \\
\hline 13 & 5 & 5005 \\
\hline 14 & 6 & 8008 \\
\hline 15 & 6 & 53068630200 \\
\hline 50 & 22 & 209895344085355160 \\
\hline 100 & 45 & \\
\hline & & 5 \\
\hline
\end{tabular}

Теорема 1. Рассмотрим произвольную $(k-1)$-мерную грань $G$ симплекса $S$. Пусть $H-(n-k)$-мерная грань $S$, которая содержит вершины, не принадлежащие $G$. Обозначим через $\mathrm{g}$ и $h$ центры тяжести граней $G$ и Н. Пусть у есть точка пересечения прямой $(\mathrm{g} h)$ с граничной сферой в направлении от $g$ к $h$. Тогда

$$
\lambda(y)=\sum_{j=1}^{n+1}\left|\lambda_{j}(y)\right|=\|P\|_{B} .
$$

Доказательство. Достаточно рассмотреть фиксированные шар $B \subset \mathbb{R}^{n}$ и правильный симплекс $S$, вписанный в $B$, а также случай $G=\operatorname{conv}\left(x^{(1)}, \ldots, x^{(k)}\right)$.

Если $n=1$, то $\psi(t)=\sqrt{t(2-t)}+|1-t|, a=0, \psi(a)=\psi(a+1)=1, k=a+1=1$. Возьмём $x^{(1)}=0$, $x^{(2)}=1$, т. е. $S=B=[0,1]$. В этом случае $g=0, h=1, \lambda_{1}(x)=-x+1, \lambda_{2}(x)=x, \lambda(x) \equiv 1$. Так как $\|P\|_{B}=1$, то при $y=h=1$ равенство (6) выполняется. Заметим, что в этом тривиальном случае совокупность точек максимума функции $\lambda(x)$ совпадает с шаром $B$. 
Пусть $n \geq 2$. Сначала заметим, что центр тяжести $c$ симплекса $S$ принадлежит отрезку $[g, h]$. Действительно, из равенств

$$
c=\frac{1}{n+1} \sum_{j=1}^{n+1} x^{(j)}, \quad g=\frac{1}{k} \sum_{j=1}^{k} x^{(j)}, \quad h=\frac{1}{n+1-k} \sum_{j=k+1}^{n+1} x^{(j)}
$$

следует, что $(n+1) c=k g+(n+1-k) h$, т.е.

$$
c=\frac{k}{n+1} g+\frac{n+1-k}{n+1} h .
$$

Для доказательства (6) достаточно указать линейный многочлен $p$, принимающий в узлах значения \pm 1 , такой что $p(y)=\|P\|_{B}$. Равенства $p\left(x^{(j)}\right)= \pm 1$ дают

$$
p(y)=\sum_{j=1}^{n+1} p\left(x^{(j)}\right) \lambda_{j}(y) \leq \lambda(y)=\sum_{j=1}^{n+1}\left|\lambda_{j}(y)\right| \leq \max _{x \in B} \sum_{j=1}^{n+1}\left|\lambda_{j}(x)\right|=\|P\|_{B} .
$$

Если $p(y)=\|P\|_{B}$, то все величины в этой цепочке совпадают, значит, $\lambda(y)=\|P\|_{B}$.

Покажем, что этим свойством обладает многочлен $p \in \Pi_{1}\left(\mathbb{R}^{n}\right)$ со значениями

$$
p\left(x^{(1)}\right)=\ldots=p\left(x^{(k)}\right)=-1, \quad p\left(x^{(k+1)}\right)=\ldots=p\left(x^{(n+1)}\right)=1 .
$$

Поскольку $p-$ линейная функция, из (7) и (9) следует, что $p(g)=-1, p(h)=1$. Применяя теперь (8), получаем

$$
p(c)=\frac{k}{n+1} p(g)+\frac{n+1-k}{n+1} p(h)=\frac{n+1-2 k}{n+1} .
$$

Центр тяжести правильного симплекса совпадает с центром шара. Так как приращение функции $p$ пропорционально расстоянию между точками, выполняется

$$
\frac{\|g-h\|}{p(g)-p(h)}=\frac{R}{p(y)-p(c)},
$$

где $R$ - радиус шара. Подставляя найденные значения, приходим к равенству

$$
p(y)=\frac{n+1-2 k}{n+1}+\frac{2 R}{\|g-h\|} .
$$

Величина последней дроби не зависит от выбора шара и вписанного в него правильного симплекса. Вычислим это значение для конкретных $S$ и $B$.

Именно в качестве $S$ возьмём правильный симплекс с вершинами

$$
x^{(1)}=e_{1}, \ldots, x^{(n)}=e_{n}, \quad x^{(n+1)}=\left(\frac{1-\sqrt{n+1}}{n}, \ldots, \frac{1-\sqrt{n+1}}{n}\right) .
$$

Длина любого ребра $S$ равна $\sqrt{2}$. Симплекс вписан в шар $B=B\left(x^{(0)} ; R\right)$, где

$$
x^{(0)}=\left(\frac{1-\sqrt{\frac{1}{n+1}}}{n}, \ldots, \frac{1-\sqrt{\frac{1}{n+1}}}{n}\right), \quad R=\sqrt{\frac{n}{n+1}} .
$$

В соответствии с (7) координаты точек $g$ и $h$ имеют вид

$$
g_{1}=\ldots=g_{k}=\frac{1}{k}, \quad g_{k+1}=\ldots=g_{n}=0,
$$




$$
\begin{gathered}
h_{1}=\ldots=h_{k}=\frac{1}{n+1-k} \cdot \frac{1-\sqrt{n+1}}{n}, \\
h_{k+1}=\ldots=h_{n+1}=\frac{1}{n+1-k} \cdot\left(1+\frac{1-\sqrt{n+1}}{n}\right) .
\end{gathered}
$$

Отсюда

$$
\|g-h\|^{2}=\left(\frac{1}{k}-\frac{1-\sqrt{n+1}}{n(n+1-k)}\right)^{2} \cdot k+\left(\frac{n+1-\sqrt{n+1}}{n(n+1-k)}\right)^{2} \cdot(n-k) .
$$

После несложных преобразований имеем

$$
\|g-h\|^{2}=\frac{n+1}{k(n+1-k)} .
$$

Итак, в рассматриваемой ситуации

$$
\frac{2 R}{\|g-h\|}=2 \sqrt{\frac{n}{n+1}} \cdot \frac{(k(n+1-k))^{\frac{1}{2}}}{\sqrt{n+1}}=\frac{2 \sqrt{n}}{n+1} \cdot(k(n+1-k))^{\frac{1}{2}} .
$$

Продолжая теперь (10), мы можем записать

$$
p(y)=\frac{n+1-2 k}{n+1}+\frac{2 R}{\|g-h\|}=1-\frac{2 k}{n+1}+\frac{2 \sqrt{n}}{n+1} \cdot(k(n+1-k))^{\frac{1}{2}} .
$$

Если $1 \leq k \leq \frac{n+1}{2}$, то последнее выражение совпадает с $\psi(k)$. Это неравенство для $k$ выполняется. Более того, $k$ совпадает с тем из чисел $a$ и $a+1$, на котором $\psi(t)$ принимает большее значение. Следовательно, $p(y)=\max \{\psi(a), \psi(a+1)\}=\|P\|_{B}$. Теорема доказана.

Теорема 2. В обозначениях предыдущей теоремы $[g, h]$ является отрезком максимальной длины, принадлежащим $S$ и параллельным вектору gh.

Доказательство. В [7] были получены формулы для вычисления длины и концов максимального отрезка заданного направления, принадлежащего симплексу. Можно выполнить соответствующие вычисления для конкретного симплекса и учесть соображения подобия. Однако проще воспользоваться следующей характеризацией максимального отрезка, доказанной в [7] (см. там леммы 1 и 2). Отрезок, принадлежащий симплексу и параллельный данному ненулевому вектору, имеет максимальную длину тогда и только тогда, когда каждая (n-1)-мерная грань симплекса содержит хотя бы один из его концов.

Пусть запись $x=\left\{\beta_{1}, \ldots, \beta_{n+1}\right\}$ означает, что точка $x$ имеет барицентрические координаты $\beta_{1}, \ldots, \beta_{n+1}$ относительно $S$. Через $G_{j}$ обозначим $(n-1)$-мерную грань симплекса, не содержащую вершину $x^{(j)}$. Для точек этой грани все барицентрические координаты неотрицательны, причём $\beta_{j}=0$. Имеем:

$$
\begin{gathered}
g=\frac{1}{k} \sum_{j=1}^{k} x^{(j)}=\left\{\frac{1}{k}, \ldots, \frac{1}{k}, 0, \ldots, 0\right\}, \\
h=\frac{1}{n+1-k} \sum_{j=k+1}^{n+1} x^{(j)}=\left\{0, \ldots, 0, \frac{1}{n+1-k}, \ldots, \frac{1}{n+1-k}\right\} .
\end{gathered}
$$

В этих равенствах число ненулевых барицентрических координат есть соответственно $k$ и $n+1-k$. Очевидно, $g \in G_{k+1}, \ldots, G_{n+1}, h \in G_{1}, \ldots, G_{k}$. Поэтому каждая $(n-1)$-мерная грань симплекса $S$ содержит конец отрезка $[g, h]$. Тем самым, этот отрезок имеет максимальную длину из всех отрезков в $S$ данного направления. Заметим, что это рассуждение подходит для любого симплекса и любого $k=1, \ldots, n$. Теорема доказана. 


\section{2. Инвариантность нормы проектора при афинном преобразовании}

В 1948 г. Ф. Джон [8] доказал, что каждое выпуклое тело в $\mathbb{R}^{n}$ содержит единственный эллипсоид максимального объёма, а также дал характеризацию тех выпуклых тел, для которых таким эллипсоидом является единичный евклидов шар $B_{n}$ (подробнее см., например, $\left.[9,10]\right)$. Из теоремы Джона следует аналогичное утверждение, которое характеризует единственный эллипсоид минимального объёма, содержащий данное выпуклое тело.

Мы будем рассматривать эллипсоид минимального объёма, содержащий данный невырожденный симплекс. Для краткости такой эллипсоид будем называть минимальным. Очевидно, что минимальный эллипсоид симплекса описан вокруг него. Центр этого эллипсоида совпадает с центром тяжести симплекса. Минимальный эллипсоид, описанный вокруг симплекса, является евклидовым шаром тогда и только тогда этот симплекс является правильным. Это эквивалентно тому, что из всех симплексов, содержащихся в шаре, максимальный объём имеет правильный симплекс, вписанный в этот шар (см., например, [11-13]).

Положим $\varkappa_{n}:=\operatorname{vol}\left(B_{n}\right)$. Через $\sigma_{n}$ обозначим объём правильного симплекса, вписанного в единичный шар $B_{n}$. Пусть $S$ - произвольный $n$-мерного симплекс и $E-$ минимальный эллипсоид этого симплекса. Если невырожденное аффинное отображение переводит $S$ в правильный симплекс, вписанный в $B_{n}$, то образ $E$ при этом отображении совпадает с $B_{n}$. Следовательно,

$$
\frac{\operatorname{vol}(E)}{\operatorname{vol}(S)}=\frac{\varkappa_{n}}{\sigma_{n}}
$$

Известно, что

$$
\begin{gathered}
\varkappa_{n}=\frac{\pi^{\frac{n}{2}}}{\Gamma\left(\frac{n}{2}+1\right)}, \quad \sigma_{n}=\frac{1}{n !} \sqrt{n+1}\left(\frac{n+1}{n}\right)^{\frac{n}{2}}, \\
\varkappa_{2 m}=\frac{\pi^{m}}{m !}, \quad \varkappa_{2 m+1}=\frac{2^{m+1} \pi^{m}}{(2 m+1) ! !}=\frac{2(m !)(4 \pi)^{m}}{(2 m+1) !}
\end{gathered}
$$

(см., например, [14, 15], [1]). Таким образом,

$$
\operatorname{vol}(E)=K_{n} \operatorname{vol}(S), \quad K_{n}:=\frac{\varkappa_{n}}{\sigma_{n}}=\frac{n !(\pi n)^{\frac{n}{2}}}{\Gamma\left(\frac{n}{2}+1\right)(n+1)^{\frac{n+1}{2}}} .
$$

Также справедливы равенства

$$
K_{2 m}=\frac{(2 m) !(2 \pi m)^{m}}{m !(2 m+1)^{m+\frac{1}{2}}}, \quad K_{2 m+1}=2^{m+\frac{1}{2}}\left(2-\frac{1}{m+1}\right)^{m+\frac{1}{2}} \pi^{m} m ! .
$$

Величина $K_{n}$ участвует в нижней оценке для нормы проектора, узлы которого принадлежат $B_{n}$. Пусть $\chi_{n}(t)$ есть стандартизованный многочлен Лежандра степени $n$ :

$$
\chi_{n}(t):=\frac{1}{2^{n} n !}\left[\left(t^{2}-1\right)^{n}\right]^{(n)} .
$$

Существует константа $C>0$, не зависящая от $n$, такая что для любого интерполяционного проектора $P: C\left(B_{n}\right) \rightarrow \Pi_{1}\left(\mathbb{R}^{n}\right)$

$$
\|P\|_{B_{n}} \geq \chi_{n}^{-1}\left(K_{n}\right)>C \sqrt{n} .
$$

Неравенства (11) установлены автором в [6]. Правая оценка верна, например, при

$$
C=\frac{\sqrt[3]{\pi}}{\sqrt{12 e} \cdot \sqrt[6]{3}}=0.2135 \ldots
$$


Пусть $S$ и $S^{\prime}$ - невырожденные симплексы в $\mathbb{R}^{n}$ с вершинами $x^{(j)}, \ldots, x^{(n+1)}$ и $y^{(1)}, \ldots, y^{(n+1)}$ соответственно. Обозначим через $\mathrm{S}$ матрицу вершин симплекса $S$, через $\mathrm{Y}-n \times(n+1)$-матрицу, $j$-й столбец которой содержит координаты вершины $y^{(j)}$. Пусть $\lambda_{1}, \ldots, \lambda_{n+1}-$ базисные многочлены Лагранжа симплекса $S$.

Лемма 1. Существует единственное аффинное преобразование $F$ пространства $\mathbb{R}^{n}$, переводящее $S$ в $S^{\prime}$, для которого $y^{(j)}=F\left(x^{(j)}\right)$. Равенство $y=F(x)$ эквивалентно каждому из соотношений

$$
\begin{aligned}
\left(\begin{array}{c}
y_{1} \\
\vdots \\
y_{n}
\end{array}\right) & =\mathrm{Y}\left(\mathrm{S}^{-1}\right)^{T}\left(\begin{array}{c}
x_{1} \\
\vdots \\
x_{n} \\
1
\end{array}\right) \\
y & =\sum_{j=1}^{n+1} \lambda_{j}(x) y^{(j)}
\end{aligned}
$$

Доказательство. Каждое невырожденное аффинное преобразование пространства $\mathbb{R}^{n}$ имеет вид $F(x)=A(x)+b$, где $A: \mathbb{R}^{n} \rightarrow \mathbb{R}^{n}-$ невырожденный линейный оператор. Пусть $\mathrm{A}=\left(a_{i j}\right)$ - матрица оператора $A$ в каноническом базисе. В координатном виде равенство $y=A(x)+b$ эквивалентно соотношению

$$
\left(\begin{array}{c}
y_{1} \\
\vdots \\
y_{n}
\end{array}\right)=\left(\begin{array}{cccc}
a_{11} & \ldots & a_{1 n} & b_{1} \\
\vdots & \vdots & \vdots & \vdots \\
a_{n 1} & \ldots & a_{n n} & b_{n}
\end{array}\right)\left(\begin{array}{c}
x_{1} \\
\vdots \\
x_{n} \\
1
\end{array}\right)
$$

Пусть М есть $n \times(n+1)$-матрица, стоящая в правой части. Условия $y^{(j)}=F\left(x^{(j)}\right)$ равносильны матричному равенству $\mathrm{Y}=\mathrm{MS}^{T}$. Следовательно,

$$
\mathbf{M}=\mathrm{Y}\left(\mathrm{S}^{T}\right)^{-1}=\mathrm{Y}\left(\mathrm{S}^{-1}\right)^{T} .
$$

Поэтому аффинное преобразование, удовлетворяющее условиям теоремы, является единственным и имеет вид (12).

Поскольку $\lambda_{j} \in \Pi_{1}\left(\mathbb{R}^{n}\right)$ и $\lambda_{j}\left(x^{(k)}\right)=\delta_{j}^{k}$, равенство (13) также задаёт аффинное преобразование $y=F(x)$, для которого $F\left(x^{(k)}\right)=y^{(k)}$. Из единственности $F$ следует, что (13) эквивалентно (12). Равносильность этих равенств можно установить и непосредственно. Перепишем (13) в координатном виде через коэффициенты многочленов $\lambda_{j}$ :

$$
\begin{gathered}
y=\sum_{j=1}^{n+1} \lambda_{j}(x) y^{(j)}=\sum_{j=1}^{n+1}\left(\sum_{k=1}^{n} l_{k j} x_{k}+l_{n+1, j}\right) y^{(j)}, \\
y_{i}=\sum_{j=1}^{n+1}\left(\sum_{k=1}^{n} l_{k j} x_{k}+l_{n+1, j}\right) y_{i}^{(j)}=\sum_{j=1}^{n+1}\left(\sum_{k=1}^{n} l_{k j} x_{k}\right) y_{i}^{(j)}+\sum_{j=1}^{n+1} l_{n+1, j} y_{i}^{(j)}= \\
=\sum_{k=1}^{n}\left(\sum_{j=1}^{n+1} y_{i}^{(j)} l_{k j}\right) x_{k}+\sum_{j=1}^{n+1} y_{i}^{(j)} l_{n+1, j} .
\end{gathered}
$$

Таким образом, (13) эквивалентно соотношениям

$$
y_{i}=\sum_{k=1}^{n} a_{i k} x_{k}+b_{i}, \quad a_{i k}=\sum_{j=1}^{n+1} y_{i}^{(j)} l_{k j}, \quad b_{i}=\sum_{j=1}^{n+1} y_{i}^{(j)} l_{n+1, j} .
$$

Поскольку $\mathrm{S}^{-1}=\left(l_{i j}\right)$, последние равенства равносильны (12). 
Отметим, что норма интерполяционного проектора инвариантна относительно невырожденного аффинного преобразования.

Теорема 3. Пусть $\Omega$ - выпуклое тело в $\mathbb{R}^{n}$, содержащее невырожденный симплекс $S, \Omega^{\prime}$ u $S^{\prime}-$ их образы при невырожденном аффином преобразовании, $P: C(\Omega) \rightarrow \Pi_{1}\left(\mathbb{R}^{n}\right), P^{\prime}: C\left(\Omega^{\prime}\right) \rightarrow \Pi_{1}\left(\mathbb{R}^{n}\right)-$ интерполяционные проекторы, узлы которых совпадают с вершинами симплексов $S$ и $S^{\prime}$ соответственно. Тогда $\|P\|_{\Omega}=\left\|P^{\prime}\right\|_{\Omega^{\prime}}$.

Доказательство. Пусть $x_{1}, \ldots, x_{n+1}-$ вершины симплекса $S$. Будем считать, что вершины симплекса $S^{\prime}$ занумерованы таким образом, что $y^{(j)}=F\left(x^{(j)}\right)$. При таком соответствии набор барицентрических координат произвольной точки $x \in \mathbb{R}^{n}$ относительно симплекса $S$ совпадает с набором барицентрических координат точки $y=F(x)$ относительно симплекса $S^{\prime}$. Это следует из равенств

$$
x=\sum_{j=1}^{n+1} \lambda_{j}(x) x^{(j)}, \quad y=\sum_{j=1}^{n+1} \lambda_{j}(x) y^{(j)},
$$

второе из которых совпадает с соотношением (13) леммы 1. Поэтому в соответствии с формулой (2), выражающей норму интерполяционного проектора через барицентрические координаты, имеем

$$
\|P\|_{\Omega}=\max \left\{\sum_{j=1}^{n+1}\left|\beta_{j}\right|: \sum_{j=1}^{n+1} \beta_{j} x^{(j)} \in \Omega, \sum_{j=1}^{n+1} \beta_{j}=1\right\}=\max \left\{\sum_{j=1}^{n+1}\left|\beta_{j}\right|: \sum_{j=1}^{n+1} \beta_{j} y^{(j)} \in \Omega^{\prime}, \sum_{j=1}^{n+1} \beta_{j}=1\right\}=\left\|P^{\prime}\right\|_{\Omega^{\prime}} .
$$

Теорема доказана.

Следствие 1. Пусть $S$ - невырожденный $n$-мерный симплекс, E- его минимальный эллипсоид, $S^{\prime}-$ правильный симплекс, вписанный в единичный шар $B_{n}$. Предположим, что $P: C(E) \rightarrow \Pi_{1}\left(\mathbb{R}^{n}\right)$ и $P^{\prime}: C\left(B_{n}\right) \rightarrow \Pi_{1}\left(\mathbb{R}^{n}\right)$ - интерполяциионые проекторы, узлы которых совпадают соответственно с вершинами $S$ и $S^{\prime}$. Тогда $\|P\|_{E}=\left\|P^{\prime}\right\|_{B_{n}}$.

Доказательство. Рассмотрим невырожденное аффинное отображение, которое переводит симплекс $S$ в правильный симплекс $S^{\prime}$. Это отображение переводит эллипсоид $E$ в шар $B_{n}$. Остаётся применить теорему 3 в ситуации, когда $\Omega$ есть минимальный эллипсоид симплекса $S$.

Дополним следствие 1 замечанием, справедливость которого устанавливается по той же схеме. Обозначим здесь через $\lambda_{j}$ базисные многочлены Лагранжа произвольного симплекса $S$. Точки минимального эллипсоида $E$, в которых достигается максимум функции $\lambda(x)=\sum\left|\lambda_{j}(x)\right|$, имеют то же геометрическое описание, которое выражается теоремой 1. В условии теоремы правильный симплекс нужно заменить произвольным, а описанный шар - минимальным эллипсоидом симплекса. В указанных точках границы эллипсоида значение $\lambda(x)$ максимально и равно $\|P\|_{E}$.

Следствие 2. Существует универсальная константа $C>0$, такая что для любого эллипсоида $E \subset \mathbb{R}^{n}$ и любого интерполяционного проектора, узлы которого принадлежат Е, выполняются неравенства $\|P\|_{E} \geq \chi_{n}^{-1}\left(K_{n}\right)>C \sqrt{n}$.

Сразу получается из (11) и предыдущего следствия.

\section{3. Об одном экстремальном свойстве правильного симплекса, вписанного в шар}

Пусть $S$ - невырожденный $n$-мерный симплекс, $E$ - минимальный эллипсоид, содержащий $S$. Зафиксируем натуральное $m \leq \frac{n}{2}$. Каждому набору из $m$ вершин симплекса поставим в соответствие точку $y \in E$, определяемую следующим образом. Пусть $g$ есть центр тяжести $(m-1)$-мерной грани $S$, содержащей выделенные вершины, $h$ - центр тяжести $(n-m)$-мерной грани, содержащей остальные 
$n+1-m$ вершин. Тогда $y$ есть точка пересечения прямой $(g h)$ с границей эллипсоида в направлении от $g$ к $h$.

Сформулируем следующую гипотезу.

(H1) Для данного натурального $m \leq \frac{n}{2}$ и любого невырожденного симплекса $S \subset B_{n}$ найдётся такой набор из т вершин симплекса, для которого $y \in B_{n}$.

Более сильный вариант гипотезы утверждает, что указанное свойство выполняется для любого натурального $m \leq \frac{n}{2}(\mathrm{H} 2)$. Для наших целей достаточно, чтобы (Н1) было справедливо для $m=k(n)$. Число $k=k(n)$ определяется в пункте 1 .

Теорема 4. Для $m=1$ гипотеза (Н1) верна.

Доказательство. Пусть $S$ - симплекс с вершинами $x^{(j)} \in B_{n}$ и центром тяжести $c$. Центр минимального для $S$ эллипсоида также находится в точке $c$. Поэтому в случае $m=1$ точки $y$ имеют вид $y^{(j)}=2 c-x^{(j)}, j=1, \ldots, n+1$. Тем самым требуется показать, что для некоторой вершины $x$ симплекса верно $\|2 c-x\| \leq 1$. Так как $S$ является невырожденным, найдётся вершина $x$, удовлетворяющая неравенству $(c, x-c) \geq 0$. Для этой вершины

$$
\|2 c-x\|^{2}=(2 c-x, 2 c-x)=4(c, c-x)+\|x\|^{2} \leq\|x\|^{2} \leq 1,
$$

Значит, вершина $x$ является подходящей. Теорема доказана.

Обозначим через $\theta_{n}\left(B_{n}\right)$ минимальную норму интерполяционного проектора $P: C\left(B_{n}\right) \rightarrow$ $\Pi_{1}\left(\mathbb{R}^{n}\right)$, узлы которого принадлежат $B_{n}$. Через $P^{\prime}$ обозначим проектор, узлы которого совпадают с вершинами правильного симплекса $S^{\prime}$, вписанного в $B_{n}$.

Теорема 5. Пусть $n$ таково, что утверждение (H1) верно для $m=k(n)$. Тогда $\theta_{n}\left(B_{n}\right)=\left\|P^{\prime}\right\|_{B_{n}}$.

Доказательство. Рассмотрим произвольный интерполяционный проектор $P$ с узлами $x^{(j)} \in B_{n}$. Пусть $S$ - симплекс с вершинами в этих точках, $\lambda_{j}-$ базисные многочлены Лагранжа для $S$. Обозначим через $E$ минимальный эллипсоид этого симплекса. Поскольку $S \subset B_{n}$, для некоторого набора из $k=k(n)$ вершин симплекса соответствующая точка $y$ принадлежит шару. Зафиксируем $y$ и запишем следующую цепочку соотношений:

$$
\left\|P^{\prime}\right\|_{B_{n}}=\|P\|_{E}=\max _{x \in E} \sum_{j=1}^{n+1}\left|\lambda_{j}(x)\right|=\sum_{j=1}^{n+1}\left|\lambda_{j}(y)\right| \leq \max _{x \in B_{n}} \sum_{j=1}^{n+1}\left|\lambda_{j}(x)\right|=\|P\|_{B_{n}} .
$$

Мы применили формулу для нормы проектора, теорему 1, следствие 1 , а также замечание после него. Неравенство в этой цепочке следует из того, что $y \in B_{n}$. Заметим, что если $y$ является внутренней точкой шара, то это неравенство является строгим.

Таким образом, для любого проектора, узлы котрого принадлежат $B_{n}$, выполняется неравенство $\left\|P^{\prime}\right\|_{B_{n}} \leq\|P\|_{B_{n}}$. Следовательно, $\theta_{n}\left(B_{n}\right)=\left\|P^{\prime}\right\|_{B_{n}}$, что и требовалось доказать.

Следствие 3. Если $1 \leq n \leq 4$, mo $\theta_{n}\left(B_{n}\right)=\left\|P^{\prime}\right\|_{B_{n}}$.

Доказательство. В случае $n=1$ утверждение эквивалентно тому, что минимальная норма интерполяционного проектора $P: C[-1,1] \rightarrow \Pi_{1}(\mathbb{R})$ реализуется для проектора, узлы которого совпадают с концами отрезка $[-1,1]$. Если же $2 \leq n \leq 4$, то выполняется равенство $k(n)=1$ и нужный результат сразу следует из теорем 4-5.

Результат следствия 3 был установлен в [2] другим методом, подходящим лишь для размерностей со свойством $k(n)=1$. Начиная с $n=5$ выполняется неравенство $k(n)>1$ (см. [2]). Однако равенство $\theta_{n}\left(B_{n}\right)=\left\|P^{\prime}\right\|_{B_{n}}$ может быть получено на пути, отмеченном теоремой 5 .

В утверждениях этого пункта единичный шар $B_{n}$ может быть заменён на произвольный евклидов шар $B$, что приведёт к эквивалентным результатам. 


\section{References}

[1] M. V. Nevskii, Geometricheskie Ocenki v Polinomial'noj Interpolyacii. Yaroslavl: P. G. Demidov Yaroslavl State University, 2012, in Russian.

[2] M. V. Nevskii and A. Y. Ukhalov, "Linear Interpolation on a Euclidean Ball in $\mathbb{R}^{n \text { ", Modeling and }}$ Analysis of Information Systems, vol. 26, no. 2, pp. 279-296, 2019. Dor: 10.18255/1818-1015-20192-279-296.

[3] M. V. Nevskii and A. Y. Ukhalov, "On Optimal Interpolation by Linear Functions on an $n$-Dimensional Cube", Modeling and Analysis of Information Systems, vol. 25, no. 3, pp. 291-311, 2018. Dor: 10.18255/ 1818-1015-2018-3-291-311.

[4] M. Nevskii and A. Ukhalov, "Perfect Simplices in $\mathbb{R}^{5 ", ~ B e i t r . ~ A l g e b r a ~ G e o m ., ~ v o l . ~ 59, ~ n o . ~ 3, ~ p p . ~ 501-521, ~}$ 2018. DOI: 10.1007/s13366-018-0386-6.

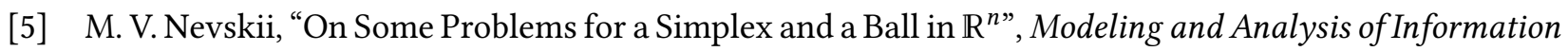
Systems, vol. 25, no. 6, pp. 680-691, 2018. DoI: 10.18255/1818-1015-2018-6-680-691.

[6] M. V. Nevskii, "Geometric Estimates in Interpolation on an n-Dimensional Ball”, Modeling and Analysis of Information Systems, vol. 26, no. 3, pp. 441-449, 2019. DoI: 10.18255/1818-1015-2019-3-441-449.

[7] M. V. Nevskii, "Computation of the Longest Segment of a Given Direction in a Simplex", fournal of Mathematical Sciences, vol. 203, no. 6, pp. 851-854, 2014.

[8] F. John, "Extremum Problems with Inequalities as Subsidiary Conditions", in Studies and essays presented to R. Courant on his 60th birthday (Jan. 8, 1948), New York: Interscience, 1948, pp. 187-204.

[9] K. Ball, Ellipsoids of Maximal Volume in Convex Bodies, arXiv: math/9201217v1 [math. MG], Sep 25, 1990.

[10] K. Ball, “An Elementary Introduction to Modern Convex Geometry”, Math. Sci. Res. Inst. Publ., vol. 31, no. 1, pp. 1-58, 1997.

[11] L. Fejes Tót, Regular Figures. New York: Macmillan/Pergamon, 1964.

[12] D. Slepian, “The Content of Some Extreme Simplices”, Pacific f. Math, vol. 31, pp. 795-808, 1969.

[13] D. Vandev, "A Minimal Volume Ellipsoid around a Simplex", C. R. Acad. Bulg. Sci., vol. 45, no. 6, pp. 37-40, 1992.

[14] G. M. Fikhtengol'ts, Kurs Differencial'nogo i Integral'nogo Ischisleniya. Tom 3. Moscow: Fizmatlit, 2001, in Russian.

[15] A. P. Prudnikov, Y. A. Brychkov, and O. I. Marichev, Integraly i Ryady. Moscow: Nauka, 2002, in Russian. 